Commentary

\title{
Commentary on "Long-term validity of the At Risk Mental State (ARMS) for predicting psychotic and non-psychotic mental disorders"
}

The management of young patients with a high risk to develop a psychotic disorder is currently one of the hot topic of psychiatry because the reduction of conversion to a full-blown psychotic disorder has an enormous advantage in terms of social functioning and well-being of these subjects. To date, if some cognitive strategies have yielded hopeful results, pharmacological approaches, including the use of antipsychotics, have not shown a clear efficacy in reducing the risk of conversion to a psychotic disorder [1]. In this sense, we read with interest the article by Fusar-Poli and co-authors about the validity of the At Risk Mental State (ARMS) criteria for future prediction of psychotic versus nonpsychotic mental conditions. The authors concluded in a quite large sample that the ARMS specifically predicts the onset of psychotic disorders, but not the risk of non-psychotic mental conditions [2]. However, Fusar-Poli and co-authors classified Bipolar Disorder (BD) as a non-psychotic condition, although till $68 \%$ of bipolar patients present lifetime psychotic symptoms. Psychotic BD shows peculiarities which differentiate this condition from both non-psychotic BD and schizophrenia [3]. As an example, treatment with mood stabilizers has demonstrated to improve outcome of psychotic bipolar patients, but not to prevent reexacerbations of subjects affected by schizophrenia [4]. In this framework we have retrospectively reconstructed how many of 235 patients (106 males and 129 females), who have regularly followed up in our outpatient clinic for at least 5 years, satisfied the ARMS criteria before the development of a full-blown BD, distinguishing between psychotic and non-psychotic subjects. The information for ARMS was taken retrospectively from the clinical charts, interviews with patients and their relatives and data collected in the Lombardy database. Thirty-four percent $(\mathrm{N}=80)$ of the total sample satisfied the ARMS criteria [5] before the onset of the first major mood episode. One hundred and seventy one subjects ( $72.8 \%$ ) had shown psychotic symptoms in at least one mood episode. Psychotic bipolar patients satisfied more frequently the ARMS criteria before the onset of $\mathrm{BD}(45.0 \%)$ as compared to non-psychotic ones $(4.7 \%) \quad(\chi 2=33.75, \mathrm{df}=1$, $\mathrm{p}<0.001$ ) (Fig. 1).

These results suggest that:

- the presence of ARMS criteria is more frequently associated with a future diagnosis of psychotic BD and this is consistent with the results of the publication by Fusar-Poli and collaborators - that ARMS criteria predict psychotic disorders;

- a large part of bipolar patients have lifetime psychotic symptoms, but BD cannot be considered a psychotic condition like schizophrenia in the light of the different associated biomarkers, specific clinical management and targeted pharmacological treatment (e.g. with lithium);

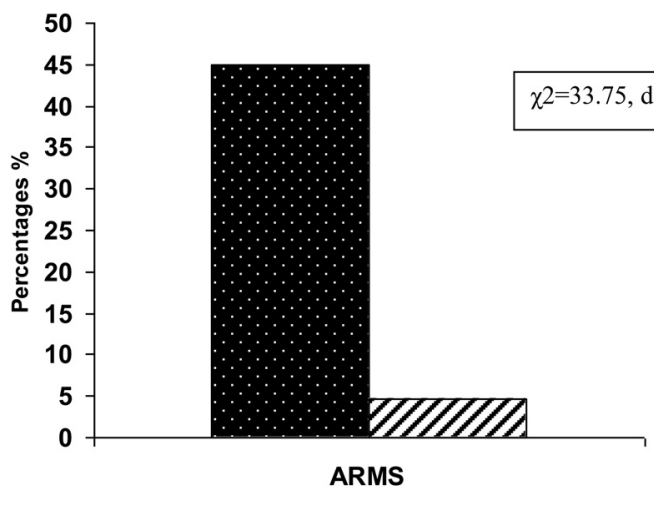

- Psychotic BD $\square$ Non-Psychotic BD

Fig. 1. Frequency of At Risk Mental State (ARMS) before the onset of a psychotic versus non-psychotic Bipolar Disorder (BD). 
- it would be useful in future research studies about this topic to consider BD as a separate category or at least to distinguish psychotic BD within the group of psychotic disorders. This latter point is extremely important because, if ARMS criteria seem to have a sort of accuracy in predicting the onset of psychotic disorders, it is not currently possible to know if high-risk subjects will develop schizophrenia or psychotic BD in case of conversion to a full-blown psychotic disorder. The category "psychotic disorders" may include an extreme heterogeneity of patients: subjects affected by schizophrenia, brief psychotic disorder, psychotic BD who need a very different management and have a distinct prognosis. This aspect could also explain the contradictory results about the effectiveness of antipsychotics in preventing conversion to psychosis in high-risk subjects. Future research will have to identify which clinical and biological markers may alternatively predict the future onset of schizophrenia or psychotic BD, and also to provide robust data about the effectiveness of mood stabilizers (e.g. lithium) for preventing conversion to a psychotic disorder in high-risk individuals.

\section{References}

[1] Yung A.R., Phillips LJ, Nelson B, Francey SM, PanYuen H, Simmons MB, et al. Randomized controlled trial of interventions for young people at ultra high risk for psychosis: 6-month analysis. J Clin Psychiatry 2011;72:430-40.
[2] Fusar-Poli P, Rutigliano G, Stahl D, Davies C, De Micheli A, Ramella-Cravaro V, et al. Long-term validity of the At Risk Mental State (ARMS) for predicting psychotic and non-psychotic mental disorders. Eur Psychiatry 2017;42:49-54.

[3] Buoli M, Caldiroli A, Cumerlato Melter C, Serati M, et al. Biological aspects and candidate biomarkers for psychotic bipolar disorder: a systematic review. Psychiatry Clin Neurosci 2016;70:227-44.

[4] Maj M, Pirozzi R, Bartoli L, Magliano L. Long-term outcome of lithium prophylaxis in bipolar disorder with mood-incongruent psychotic features: a prospective study. J Affect Disord 2002;71:195-8.

[5] Yung AR, Nelson B. The ultra-high risk concept-a review. Can J Psychiatry 2013;58:5-12.

Massimiliano Buoli* Cecilia Maria Esposito Alice Caldiroli

Department of Psychiatry, University of Milan, Fondazione IRCCS Ca' Granda Ospedale Maggiore Policlinico, Milan, Italy

* Corresponding author at: Department of Psychiatry, University of Milan, Fondazione IRCCS Ca' Granda Ospedale Maggiore Policlinico, Via F. Sforza 35, 20122, Milan, Italy. E-mail address: massimiliano.buoli@unimi.it (M. Buoli).

Received 19 July 2018

Available online 24 August 2018 